\title{
In late pregnancy insulin-dependent glucose transport/ phosphorylation is selectively impaired and activation of glycogen synthase by insulin facilitated in skeletal muscles of 24-h starved rats
}

\author{
M.J. Holness, M. C. Sugden \\ Molecular and Cellular Biology, Division of Biomedical Sciences, St Bartholomew's and the Royal London School of Medicine \\ and Dentistry, Queen Mary and Westfield College, University of London, London, UK
}

\begin{abstract}
Aims/hypothesis. We investigated the relation between glucose transport/phosphorylation and glycogen synthase activation in individual rat skeletal muscles in response to moderate hyperinsulinaemia in the absence or presence of hyperglycaemia during pregnancy.

Methods. Rats were studied on day 20 of pregnancy after 24-h starvation, with unmated rats as controls. Insulin and glucose were infused into conscious rats through an indwelling cannula as specified. Glucose transport/phosphorylation was assessed in vivo using 2-deoxy-[1- $\left.{ }^{3} \mathrm{H}\right]$ glucose. Glycogen synthase activity was measured in muscle extracts $\pm 10 \mathrm{mmol} / \mathrm{l}$ glucose-6-phosphate.

Results. In unmated rats, stimulation of glucose transport/phosphorylation occurred in response to euglycaemic-hyperinsulinaemia in all muscles studied but activation of glycogen synthase was not observed. Muscle glucose transport/phosphorylation rates during euglycaemic-hyperinsulinaemia after 24-h starvation were lower in pregnant compared with unmated rats, whereas glycogen synthase activation by insulin
\end{abstract}

occurred in two of three fast-twitch muscles of pregnant rats. When insulin and glucose concentrations were matched between the 24-h starved unmated and pregnant groups through variable glucose infusion $(15 \mathrm{~min})$, glycogen synthase activities in fasttwitch muscles did not differ between unmated and pregnant groups. The response of glycogen synthase to hyperglycaemia in slow-twitch muscle was, however, greater in the pregnant group.

Conclusion/interpretation. Glycogen synthase activation in slow-twitch muscle in response to hyperinsulinaemia after 24-h starvation is enhanced in late pregnancy but the response is critically dependent on glycaemia. Pregnancy is, nevertheless, associated with reduced muscle glucose transport/phosphorylation. This disassociation ensures that available glucose is directed towards the fetus rather than the mother under conditions of moderate hyperinsulinaemia, for example during refeeding after starvation. [Diabetologia (1999) 42: 802-811]

Keywords Glycogen synthase, skeletal muscle, pregnancy, insulin resistance.
Received: 29 June 1998 and in final revised form: 28 December 1998

Corresponding author: M. Sugden, Molecular and Cellular Biology, Division of Biomedical Sciences, Queen Mary and Westfield College, Mile End Road, London E1 4NS, UK Abbreviations: ADL, Adductor longus; AT, anterior tibialis; EDL, extensor digitorum longus; G6P, glucose-6-phosphate; GIR, glucose infusion rate; GLUT, glucose transporter; GSa, G6P-independent form of glycogen synthase; GUI, glucose utilisation index; NEFA, non-esterified fatty acid; SOL, soleus; TAG, triacylglycerol; WG, white gastrocnemius.
Glucose sparing during pregnancy is achieved through suppression of glucose utilisation by the skeletal muscle mass [1-4]. This adaptation spares glucose for use by the feto-placental unit by limiting maternal glucose utilisation $[2,3,5]$ and is also an important adaptation for safeguarding fetal nutrient supply during periods of nutrient (e.g. glucose) scarcity. Individual skeletal muscle types respond selectively to the requirement for glucose sparing during pregnancy [1]. Although suppression of glucose utilisation is more pronounced in slow-twitch (oxidative) skeletal 
muscles than in fast-twitch skeletal muscles, it is observed earlier during gestation and is proportionately greater in the fast-twitch skeletal muscles [1]. Similarly, glucose utilisation (uptake/phosphorylation) by the fast-twitch skeletal muscles of late-pregnant rats is more impaired than that of slow-twitch skeletal muscles during the initial phase of refeeding after prolonged starvation $[6,7]$. Insulin resistance is also a major characteristic of late pregnancy $[2,3,5]$. The major fate of glucose in fast-twitch skeletal muscles under hyperinsulinaemic conditions is normally storage as glycogen $[8,11]$. Despite lower rates of glucose uptake/phosphorylation in fast-twitch muscle during the initial phase of refeeding after starvation in late pregnant compared with unmated rats, there is no indication of impaired muscle glycogen storage $[6,7]$. This response optimises storage of glucose as muscle glycogen during refeeding after starvation, and may also prevent deleterious effects of hyperglycaemia on the fetus. The mechanism whereby muscle glycogen deposition is maintained despite insulin resistance at the level of glucose uptake/phosphorylation has not been resolved.

Increases in glucose transport/hexokinase activity and dephosphorylation (activation) of glycogen synthase are potentially important as determinants of glycogen synthesis during hyperinsulinaemia [11, 12]. Activation of glycogen synthase through dephosphorylation results in a reduced dependence on glucose-6-phosphate (G6P) for activity [13]. During the early phase of refeeding after starvation, both glucose uptake/phosphorylation rates and glycogen synthase phosphorylation state contribute to the regulation of glycogen synthesis; however, later there is a shift to control based solely upon changes in glycogen synthase phosphorylation state [14]. Our study was designed to investigate the mechanism by which muscle glycogen storage is protected during refeeding after starvation in late pregnancy. In particular, we focussed on the possibility that there is a shift in the control site for skeletal-muscle glycogen synthesis from glucose transport/phosphorylation towards phosphorylation state-based control of glycogen synthase. We examined the relation between glucose transport/phosphorylation and glycogen synthase phosphorylation status (as inferred from the proportion of total glycogen synthase existing in the G6P-independent form) in vivo using the euglycaemic-hyperinsulinaemic clamp technique in unmated and late-pregnant $24-\mathrm{h}$ starved rats to delineate the precise involvement of insulin in the response. These studies were conducted over a timescale $(2 \mathrm{~h})$ similar to the initial phase of refeeding after starvation. In addition, we defined the pattern of changes in the phosphorylation state of glycogen synthase after manipulation of circulating glucose concentrations through short-term glucose infusion after 24-h starvation.

\section{Materials and methods}

Reagents. Glucose assay kits were obtained from Boehringer Mannheim, Lewes, East Sussex, UK. Kits for determination of plasma insulin concentrations were from Phadeseph Pharmacia, Uppsala, Sweden. WAKO kits for determination of plasma non-esterified fatty acid (NEFA) and triacylglycerol (TAG) concentrations were from Alpha Labs, Eastleigh, Hants, UK. Other biochemicals and chemicals were from Boehringer Mannheim, or from Sigma Chemical Corporation, Poole, Dorset, UK. Radiochemicals were from Amersham International, Amersham, Bucks, UK. Female Wistar rats were purchased from Charles River Ltd., Margate, Kent, UK.

Animals. The procedures with the animals were reviewed and approved by the Home Office and were carried out in accordance with the Animals (Scientific procedures) Act 1986. Female Wistar rats were housed at $21 \pm 2{ }^{\circ} \mathrm{C}$ with controlled lighting on a $12 \mathrm{~h} \mathrm{light} / 12 \mathrm{~h}$ dark lighting cycle (light from 0800 hours). Rats were maintained on standard rodent highcarbohydrate/low-fat laboratory diet. Water was provided ad libitum. Pregnant rats were time-mated [15] and studied on day 20 of pregnancy (term is 22-23 days), with age-matched, unmated rats as controls. Pregnant and unmated rats were each fitted with two indwelling jugular cannulae (for infusion and sampling, respectively) under Hypnorm [fentanyl citrate $(0.315 \mathrm{mg} / \mathrm{ml}) /$ fluanisone $(10 \mathrm{mg} / \mathrm{ml}) ; 1 \mathrm{ml} / \mathrm{kg}$ i.p.] and Diazepam $(5-10 \mathrm{mg} / \mathrm{ml} ; 1 \mathrm{ml} / \mathrm{kg}$ i.p.) anaesthesia at 5-7 days before study. Food was withdrawn at the end of the dark (feeding) phase at 0800 hours and rats were studied at $24 \mathrm{~h}$ after food withdrawal. All manipulations in vivo were undertaken using conscious, unrestrained, unstressed rats. The fibre compositions (fast-twitch oxidative-glycolytic: fast-twitch glycolytic: slow-twitch oxidative) of the skeletal muscles studied as taken from a previous report [16], are as follows: white gastrocnemius (WG), containing mainly fast-twitch glycolytic fibres (37:58:5); anterior tibialis, (AT) and extensor digitorum longus (EDL), which contain a mixture of fast-twitch glycolytic fibres (66:32:2 and 59:38:3, respectively); adductor longus (ADL), containing mainly slow-twitch fibres but also some fast-twitch fibres (12:0:88); and soleus (SOL), containing slow-twitch fibres $(0: 0: 100)$.

Euglycaemic-hyperinsulinaemic clamps. For euglycaemic-hyperinsulinaemic clamp studies, a primed continuous infusion of insulin (Human Actrapid Insulin, NovoNordisk, Bagsvaerd, Denmark) was given at a fixed dose of $4.2 \mathrm{mU} / \mathrm{kg}$ per min for $120 \mathrm{~min}$. To maintain euglycaemia, blood glucose was clamped using a variable rate of $30 \%$ glucose infusion, which was initiated at $1 \mathrm{~min}$ after the start of insulin infusion. Blood was sampled from the right jugular vein at approximately 5-10 min intervals and blood glucose concentrations determined. Adjustments in the exogenous glucose infusion rates were made to maintain glycaemia at the target concentration $(\sim 4 \mathrm{mmol} / \mathrm{l})$. A plateau for the exogenous glucose infusion rate was reached after 60-90 min. The glucose infusion rate required to maintain glycaemia during the plateau phase of the clamp is denoted as GIR.

Glucose transport/phosphorylation studies. Glucose transport/ phosphorylation rates (glucose utilisation indices, GUI values) were estimated in maternal skeletal muscles and in fetuses at steady-state glucose concentrations in the basal (24-h starved) state or during steady-state hyperinsulinaemia from the accumulation of 2-deoxy-[1- $\left.{ }^{3} \mathrm{H}\right] \mathrm{G} 6 \mathrm{P}$ in the tissue after the bolus injection of tracer amounts of the glucose analogue 2-deoxy-[1$\left.{ }^{3} \mathrm{H}\right]$ glucose [17]. Since 2-deoxy-[1-3 H]G6P is the terminal me- 
Table 1. Blood glucose and plasma insulin concentrations in the basal (24-h starved) state and after insulin and glucose infusion rates during hyperinsulinaemia in unmated and 20-day-pregnant rats

\begin{tabular}{|c|c|c|c|c|}
\hline & \multicolumn{2}{|c|}{ Basal (24-h starved) state } & \multicolumn{2}{|c|}{ Euglycaemic-hyperinsulinaemic clamp } \\
\hline & Unmated & 20-day Pregnant & Unmated & 20-day Pregnant \\
\hline Blood glucose $(\mathrm{mmol} / \mathrm{l})$ & $3.1 \pm 0.2(5)$ & $2.2 \pm 0.2^{\mathrm{b}}(5)$ & $4.2 \pm 0.3(7)$ & $4.2 \pm 0.2(5)$ \\
\hline GIR (mg/min per rat) & - & - & $6.7 \pm 0.8(5)$ & $5.7 \pm 0.4(5)$ \\
\hline GIR (mg/min per kg body weight) & - & - & $26.4 \pm 1.8(5)$ & $16.8 \pm 1.5^{\mathrm{a}}(5)$ \\
\hline
\end{tabular}

Further details are provided in Materials and methods. Data are means \pm SEM. Numbers of observations (individual rats) are given in parentheses. Statistically significant differences between unmated and 20-day-pregnant rats are indicated by: ${ }^{\mathrm{a}} p<0.05 ;{ }^{\mathrm{b}} p<0.01$ tabolite of 2-deoxy-[1- $\left.{ }^{3} \mathrm{H}\right]$ glucose, the accumulation of 2deoxy- $\left[1-{ }^{3} \mathrm{H}\right] \mathrm{G} 6 \mathrm{P}$ provides an index of the rate of G6P generation from blood-borne glucose [18]. Rats were injected with a bolus of tracer amounts $(1.11 \mathrm{MBq})$ of the glucose analogue 2-deoxy- $\left[1-{ }^{3} \mathrm{H}\right]$ glucose in the basal state or during hyperinsulinaemia (at 90 min after the start of insulin infusion). Blood samples $(100 \mu \mathrm{l})$ for determination of blood glucose concentrations and plasma tracer concentrations were obtained at 1, 3, 5, $10,20,40$, and $60 \mathrm{~min}$ after 2 -deoxy-[1- $\left.{ }^{3} \mathrm{H}\right]$ glucose bolus injection. Throughout the study, the rats were awake and allowed to move freely, with the connecting tubing suspended overhead. At the end of the 60-min study, rats were injected intravenously with pentobarbitone $(60 \mathrm{mg} / \mathrm{kg}$ i. v.) through the right jugular cannula. Death occurred within $5 \mathrm{~s}$. Tissue samples (maternal skeletal muscles and 3 whole fetuses per dam) were rapidly removed, freeze-clamped and stored in liquid $\mathrm{N}_{2}$ until subsequent analysis $[4,17,18]$.

Glucose infusion. Glucose was given and blood withdrawn through long-term indwelling cannulae, fitted as described under the heading Animals previously [19]. Glucose solution $(50 \% \mathrm{w} / \mathrm{v})$ was infused at a priming dose of $200 \mu \mathrm{l} / \mathrm{min}$ for 20 $\mathrm{s}$, followed by variable infusion as specified to achieve sustained hyperglycaemia. Samples of whole blood $(150 \mu \mathrm{l})$, obtained at the time points indicated, were de-proteinized with $\mathrm{ZnSO}_{4} / \mathrm{Ba}(\mathrm{OH})_{2}$, centrifuged $(10000 \mathrm{~g})$ at $4{ }^{\circ} \mathrm{C}$, and the supernatant retained for subsequent assay of blood glucose. The remaining sample was immediately centrifuged $(10000 \mathrm{~g})$ at $4^{\circ} \mathrm{C}$ and plasma was stored at $-20^{\circ} \mathrm{C}$ until assayed for insulin NEFA and TAG.

Glycogen synthase assays. At the end of the glucose and insulin infusions and at intervals after bolus glucose injection, rats were anaesthetised with pentobarbitone $(60 \mathrm{mg} / \mathrm{kg}$ i.v.). After locomotor activity had ceased, skeletal muscles were excised rapidly and freeze-clamped in liquid $\mathrm{N}_{2}$. Freeze-clamped skeletal muscles were extracted for glycogen synthase assay as described previously [6]. In brief, skeletal muscle samples were homogenised (Polytron Tissue Homogeniser, Kinematica, Luzern, Switzerland) in ice-cold buffer containing $50 \mathrm{mmol} / \mathrm{l}$ potassium phosphate, $2 \mathrm{mmol} / \mathrm{l}$ dithiothreitol, $2 \mathrm{mmol} / \mathrm{l}$ EDTA, $20 \mathrm{mmol} / \mathrm{l}$ sodium fluoride (to inhibit phosphatase activity), $\mathrm{pH}$ 6.8. The extract was centrifuged for $20 \mathrm{~s}$ at $10000 \mathrm{~g}$ and the supernatant, which contains more than $95 \%$ of the glycogen synthase activity, was removed and retained on ice for subsequent glycogen synthase assay. Glycogen synthase was determined by a modification [20] of a previously reported method [21] in the absence (GSa) or presence of $10 \mathrm{mmol} / \mathrm{l}$ of the allosteric activator G6P and with $4.4 \mathrm{mmol} / \mathrm{l}$ of the substrate $\left[{ }^{14} \mathrm{C}\right]$ uridine diphosphoglucose. The percentage of glycogen synthase in the active form (\% GSa) is defined as the ratio of the activities $\pm \mathrm{G} 6 \mathrm{P} \times 100$. Protein was assayed using the Biuret method.
Hormone and metabolite assays. Blood glucose concentrations during infusion studies were determined using a glucose analyser (YSI, Yellow Springs, Ohio, USA). In other studies, blood glucose concentrations were measured in deproteinised samples by the glucose oxidase method with kits supplied by Boehringer Mannheim. Plasma insulin concentrations were measured by radioimunoassay using a kit from Phadeseph Pharmacia. Concentrations of the ketone bodies (3-hydroxybutyrate + acetoacetate) and lactate were determined in $\mathrm{KOH}$-neutralised $\mathrm{HClO}_{4}$-extracts of whole blood using enzymatic spectrophotometric methods (see [3] for details of methods). Blood was sampled in the basal (24-h starved) state or at the end of infusion studies and $\mathrm{HClO}_{4}$ extracts of whole blood were stored at $-20^{\circ} \mathrm{C}$ for later analysis within 1 week. These extracts were neutralised using $\mathrm{KOH}$ and ketone body and lactate concentrations were assayed spectrophotometrically on the same day. Plasma NEFA and TAG concentrations were measured by spectrophotometric methods using WAKO kits according to the instructions of the manufacturer.

Statistics. Results are presented as means \pm SEM. Statistical comparisons were made with StatView (Abacus Concepts, Inc., Berkeley, Calif., USA). Statistical analysis was by analysis of variance, followed by a post hoc Fisher's test, or by Student's $t$ test for single comparisons.

\section{Results}

Skeletal muscle glucose utilisation after 24-h starvation in unmated and late-pregnant rats. Steady-state blood glucose and plasma insulin concentrations measured in unmated and 20-day-pregnant rats after 24-h starvation (basal state) are shown in Table 1. Blood glucose concentrations were lower $(p<0.01)$ in the late-pregnant rats but plasma insulin concentrations did not differ significantly between the groups. The GUI values after 24-h starvation were lower in late-pregnant rats compared with unmated rats in the three skeletal muscles containing predominantly fast-twitch muscle fibres [WG (by $65 \%$; $p<0.001$ ), EDL (by $60 \% ; p<0.001$ ), and AT (by $65 \% ; p<0.01$ )] (Fig. 1). Similarly, GUI values in the basal state were lower in late-pregnant rats compared with unmated rats in the two skeletal muscles containing mainly slow-twitch fibres [ADL (60\%; $p<0.05)$ and SOL $(39 \% ; p<0.01)]$. 


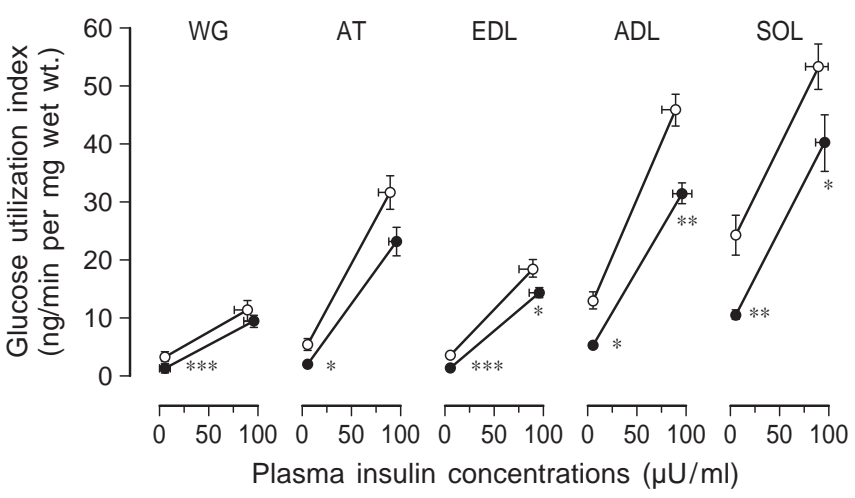

Fig.1. Effects of late pregnancy on glucose utilisation in individual skeletal muscles in the basal state and after euglycaemic insulin stimulation in unmated and 20-day-pregnant rats. Glucose utilisation indices values in WG, AT, EDL, SOL and ADL of unmated (open symbols) and 20-day-pregnant rats (closed symbols) were measured in the basal (24-h starved) state and after $120 \mathrm{~min}$ of euglycaemic hyperinsulinaemia. Results for each muscle have been plotted as a function of the prevailing insulin concentration. Further details are provided in Materials and methods. Glucose utilisation indices values were measured in both muscles for individual rats. The results represent means \pm SEM for 6 unmated and 5 late-pregnant rats in the basal state and for 7 unmated and 5 late-pregnant rats in the insulin-stimulated state. Statistically significant differences between unmated and 20-day-pregnant rats in either the basal or hyperinsulinaemic states are indicated by $* p<0.05 ; * * p<0.01 ; * * * p<0.001$. Statistical analysis of the effects of hyperinsulinaemia is provided in the text

Whole-body glucose utilisation during insulin stimulation after 24-h starvation. Steady-state insulin concentrations during the euglycaemic-hyperinsulinaemic clamps were raised to $35-40 \mu \mathrm{U} / \mathrm{ml}$ (Table 1 ). These insulin concentrations are within the range of concentrations observed during free feeding [22]. To facilitate direct comparison of whole-body insulin action between the two groups, blood glucose concentrations during insulin infusion were standardised to approximately $4 \mathrm{mmol} / \mathrm{l}$, the blood glucose concentration found in 20-day-pregnant rats in the fed (absorptive) phase [1, 3]. At matched glucose and insulin concentrations (Table 1 ), the GIR required to maintain glycaemia, expressed on a whole rat basis, was approximately $15 \%$ lower in the pregnant rats, despite their much greater body weights. The GIR (expressed on a $\mathrm{kg}$ body weight basis) was $30 \%(p<0.01)$ lower in the pregnant group, confirming the existence of whole-body insulin resistance in late pregnancy $[2,3,5]$. The reduced requirement for glucose to maintain glycaemia in the pregnant group may reflect the presence of maternal and fetal tissues that are relatively insulin non-responsive during pregnancy. In our experiments, the mean mass of each fetus at day 20 of pregnancy was $3.665 \pm 0.012 \mathrm{~g}(n=5)$. Estimates of fetal glucose utilisation using 2 -deoxy- $\left[1-{ }^{3} \mathrm{H}\right]$ glucose suggested a total fetal rate of glucose utilisation of approxi- mately $0.4 \mathrm{mg} / \mathrm{rat}$ per min $(7 \%$ of the whole body glucose requirement).

Skeletal muscle glucose utilisation during insulin stimulation. In unmated rats, GUI values in the three skeletal muscles containing predominantly fast-twitch fibres, WG, AT and EDL, increased significantly (by 5.9-fold, 3.6-fold and 5.3-fold, respectively; $p<0.001$ ) in response to hyperinsulinaemia (Fig.1). Similarly, GUI values for the two skeletal muscles containing predominantly slow-twitch muscle fibres, namely SOL and ADL, increased (by 2.2-fold and 3.5-fold, respectively; $p<0.001$ ) in response to hyperinsulinaemia in unmated rats (Fig.1). Muscle GUI values also increased $(p<0.001)$ in response to hyperinsulinaemia in the late-pregnant rats (Fig.1). Nevertheless, GUI values during hyperinsulinaemia were lower in EDL (by $23 \% ; p<0.05$ ), ADL (by $31 \% ; p<0.01$ ) and SOL (by $24 \% ; p<0.05$ ) in muscles of late-pregnant rats compared with those of the unmated controls (Fig.1). Furthermore, in the pregnant group, the increment in GUI value elicited in response to hyperinsulinaemia was lower in several of the skeletal muscles studied, namely AT (by 19\%), EDL (by 14\%) and ADL (by $20 \%$ ). This parallels the reduction in the glucose requirement for maintenance of glycaemia during hyperinsulinaemia, supporting previous studies indicating that skeletal muscle contributes to wholebody insulin resistance during late pregnancy $[2,3,5]$.

Muscle glycogen concentrations. There is a strong inverse relation between tissue net glycogen accumulation and tissue glycogen content during insulin stimulation after glycogen depletion [23, 24]. This is consistent with a dominant role for the glycogen mass in the regulation of glycogen repletion and is possibly a consequence of the regulatory effect of the prevailing glycogen concentration on glycogen synthase activity [25]. Skeletal-muscle glycogen concentrations in the fed state and after 24-h starvation are shown for unmated and late-pregnant rats in Figure 2. Glycogen concentrations in four of the five muscles studied did not differ significantly between unmated and latepregnant rats in the fed state. In the fifth muscle (EDL), glycogen concentrations were higher (by $8 \%$, $p<0.05)$ in the pregnant group in the fed state. Starvation for $24 \mathrm{~h}$ was associated with depletion of skeletalmuscle glycogen stores both in unmated and in latepregnant rats (Fig.2). The statistical significance of muscle glycogen loss was, with the exception of ADL, greater in the pregnant compared with the unmated group, but there were no significant differences in skeletal-muscle glycogen concentrations between unmated and late-pregnant rats after 24-h starvation (Fig. 2).

Muscle glycogen synthase activities in the basal state and at the end of the euglycaemic hyperinsulinaemic clamps. Consistent with a previous study [26], mean 


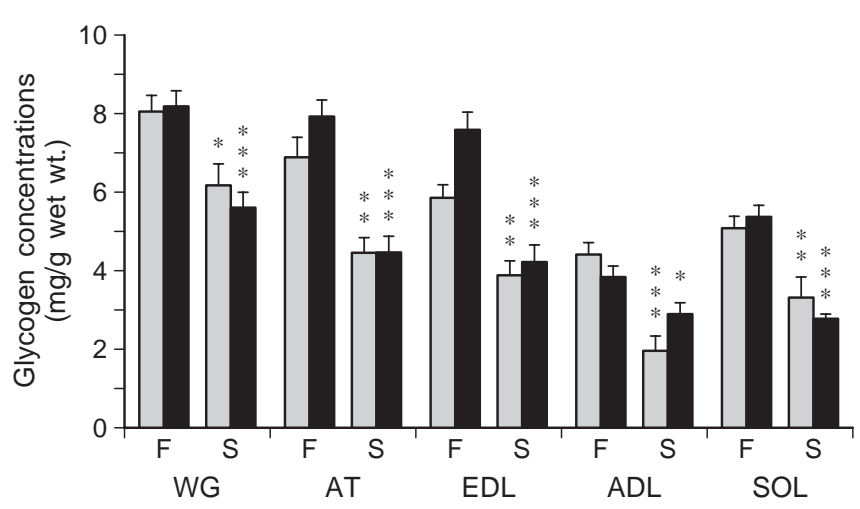

Fig. 2. Effects of late pregnancy on glycogen concentrations in individual skeletal muscles in the fed and 24-h starved states in unmated and 20-day-pregnant rats. Glycogen concentrations in WG, AT, EDL, SOL and ADL of unmated (grey bars) and 20-day-pregnant rats (black bars) were measured in the fed state (at the end of the dark phase) and after 24-h starvation. Further details are provided in Materials and methods. Results are means \pm SEM for 17 unmated and 17 pregnant rats in the fed state and for 6 unmated and 9 late-pregnant rats in the 24h starved state. Statistically significant effects of starvation on glycogen concentrations in either unmated or 20-day-pregnant rats are indicated by * $p<0.05$; ** $p<0.01$; *** $p<0.001$, $\mathrm{F}=$ fed, $\mathrm{S}=$ after 24 -h starvation. Statistical analysis of the effects of late pregnancy is provided in the text

Table 2. Total GS activities for individual skeletal muscles of unmated and 20-day-pregnant rats

\begin{tabular}{lll}
\hline & \multicolumn{2}{l}{ Total GS activity } \\
\cline { 2 - 3 } & Unmated & 20-day-pregnant \\
\hline Gastrocnemius & $224 \pm 23(16)$ & $221 \pm 14(19)$ \\
Anterior tibialis & $301 \pm 31(16)$ & $293 \pm 20(19)$ \\
Extensor digitorum longus & $231 \pm 19(16)$ & $216 \pm 14(19)$ \\
Soleus & $284 \pm 22(16)$ & $275 \pm 12(19)$ \\
Adductor longus & $225 \pm 23(16)$ & $208 \pm 14(19)$ \\
\hline
\end{tabular}

Values for total GS activity ${ }^{\text {a }}$ (sum of G6P-dependent and G6Pindependent forms, measured in the presence of $10 \mathrm{mmol} / \mathrm{l}$ G6P) are expressed as $\mathrm{nmol} / \mathrm{h}$ per $\mathrm{mg}$ protein and are the means of values obtained for individual skeletal muscles of rats sampled in the basal (24-h starved) state. There were no statistically significant effects of late pregnancy on total GS activities for individual skeletal muscles. Further details are provided in Materials and methods. Data are means \pm SEM. Numbers of observations (rats) are given in parentheses

total glycogen synthase activities (measured in the presence of $10 \mathrm{mmol} / \mathrm{l} \mathrm{G6P}$ ) in the range of skeletal muscles studied did not differ between unmated and late-pregnant rats (Table 2). Despite lower rates of glucose transport/phosphorylation, the per cent of G6P-independent form of glycogen synthase (\%GSa) present in the skeletal muscles after 24-h starvation was not diminished in late-pregnant rats and, indeed, was increased $(p<0.05)$ in AT (Fig. 3). Whereas $120 \mathrm{~min}$ of euglycaemic hyperinsulinaemia did not significantly increase \%GSa in any of the five skeletal muscles studied in the unmated group, increases in the \%GSa were observed in AT $(p<0.05)$ and EDL $(p<0.01)$ of the pregnant group (Fig. 3). Values of \%GSa in AT, but not WG or EDL, at the end of the infusions were higher $(p<0.05)$ in the pregnant than in the unmated rats. Values of \%GSa in the two slow-twitch muscles at the end of the infusions did not differ significantly between pregnant and unmated rats. There was no impairment in the increment in muscle \%GSa elicited by hyperinsulinaemia between unmated and pregnant 24-h starved rats. In fact, in fast-twitch muscles the increment in muscle \% GSa elicited by hyperinsulinaemia was 2.2-fold to 2.9-fold higher in pregnant compared with unmated rats although this did not achieve statistical significance.

Muscle glycogen synthase activities after glucose infusions. Glucose was infused into 24-h starved rats to induce endogenous insulin secretion in response to hyperglycaemia. Glucose and insulin concentrations are shown in Figure 4. In both unmated and pregnant 24-h starved rats, glucose infusion led to increases in blood glucose concentrations within $5 \mathrm{~min}$ of the start of the infusions (unmated, 2.4-fold, $p<0.01$; pregnant, 3.0-fold, $p<0.01$ ). Plasma insulin concentrations were raised after 10 and 15 min of glucose infusion both in unmated $(p<0.05)$ and late-pregnant $(p<0.01)$ 24-h starved rats. Glucose and insulin concentrations at 10 and 15 min after the start of the infusions did not differ significantly between unmated and late-pregnant rats (Fig. 4). Muscle \% GSa activities were measured after glucose infusion for $15 \mathrm{~min}$ (i.e. after exposure to increased glucose and insulin concentrations for at least $5 \mathrm{~min}$ ). After $15 \mathrm{~min}$ of glucose infusion, the \%GSa existing in muscles of unmated rats starved previously for $24 \mathrm{~h}$ had increased in WG, AT and EDL (to $\approx 1.4$-fold to 2.0-fold of basal); non-significant trends towards increased \%GSa were observed in SOL and ADL (Fig. 5; [26]). In the 24-h starved pregnant group, the exposure to increased glucose and insulin concentrations increased \%GSa in four of the five muscles studied, including both of the slow-twitch skeletal muscles (Fig. 5). In two of the three fast-twitch skeletal muscles (WG and AT), the increment in \% GSa elicited by glucose infusion were less $(p<0.05)$ in 24-h starved pregnant rats than in the 24-h starved unmated controls. This reflected the higher basal $\%$ GSa observed in WG and AT in the late-pregnant rats. Nevertheless, the \%GSa existing in WG, AT and EDL at the end of the 15-min period of glucose infusion did not differ significantly between unmated and late-pregnant rats. In contrast, the response of glycogen synthase to glucose infusion in the two slow-twitch muscles, ADL and SOL, was considerably enhanced in 24-h starved, late-pregnant rats (Fig. 5). The increment in \% GSa elicited by hyperinsulinaemia was 3.7-fold $(p<0.01)$ higher in SOL and 


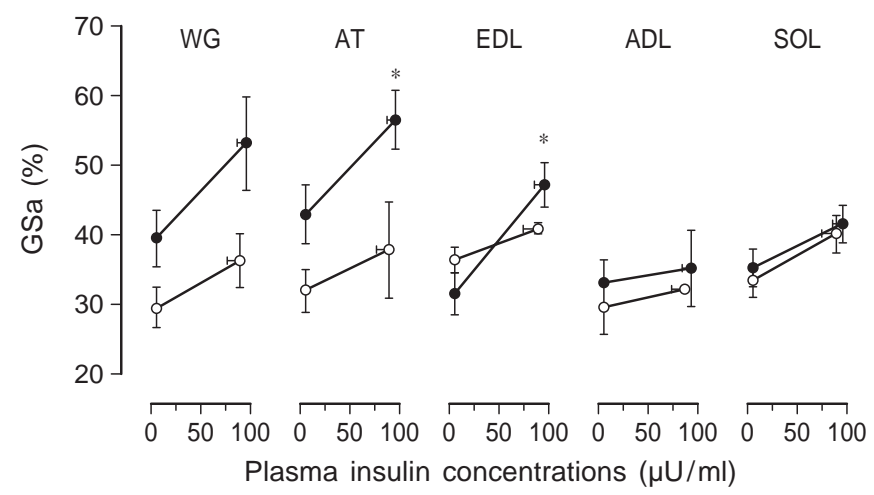

Fig. 3. Effects of euglycaemic hyperinsulinaemia on G6P-independent glycogen synthase activities in individual skeletal muscles of unmated and 20-day-pregnant rats. The G6P-dependent and G6P-independent glycogen synthase activities were measured in WG, AT, EDL, SOL and ADL of unmated (open symbols) and 20-day-pregnant rats (closed symbols) in the basal (24-h starved) state and after 120 min euglycaemic hyperinsulinaemia. The \%GSa values for each muscle are plotted as a function of the prevailing insulin concentration. Further details are provided in Materials and methods. Results are means \pm SEM for 7 unmated and 7 late-pregnant rats in the basal state and for 5 unmated and 6 late-pregnant rats in the insulin-stimulated state. Statistically significant effects of hyperinsulinaemia in either unmated and 20-day-pregnant rats are indicated by $* p<0.05$. Statistical analysis of the differences between unmated and 20-day-pregnant rats in either the basal or hyperinsulinaemic states is provided in the text
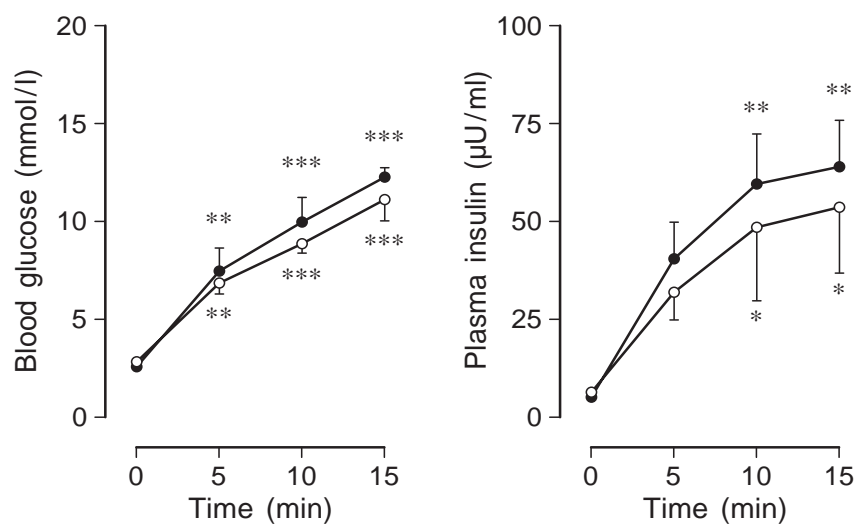

Fig.4. Effects of intravenous glucose infusion on blood glucose and plasma insulin concentrations in unmated rats (open symbols) and 20-day-pregnant rats (closed symbols). Glucose solution $(50 \%, \mathrm{w} / \mathrm{v})$ was infused as described in Materials and methods. Results are means \pm SEM for 6 separate animals in each group. Statistically significant differences between values before and after glucose infusion are indicated by: $* p<0.05$; $* * p<0.01$; *** $p<0.001$. There were no statistically significant differences between unmated and 20-day-pregnant rats at any of the points in time

1.6-fold (NS) higher in ADL muscles of pregnant compared with unmated rats. As a consequence, the $\%$ GSa existing in the two slow-twitch muscles at the end of the 15-min glucose infusion was higher [by $77 \%$ in SOL $(p<0.001)$ and $36 \%$ in ADL $(\mathrm{NS})]$ in

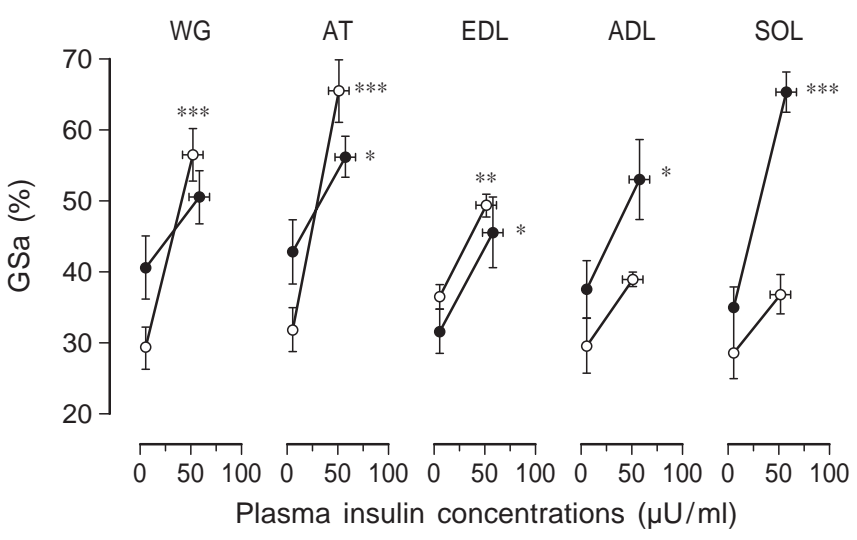

Fig. 5. The response of G6P-independent glycogen synthase activity to glucose infusion in individual skeletal muscles of unmated and 20-day-pregnant rats. The \% GSa in WG, AT, EDL, SOL and ADL were measured in the basal (24-h starved) state and after $15 \mathrm{~min}$ of variable glucose infusion for unmated rats after (open symbols) and 20-day-pregnant rats (closed symbols). Further details are provided in Materials and methods. Results are means \pm SEM for 6 rats in each group in the basal state and for 4 (unmated) or 8 (pregnant) rats in the glucose-infused groups. Statistically significant effects of glucose infusion on \% GSa in either unmated or 20-day-pregnant rats are indicated by: $* p<0.05 ; * * p<0.01 ; * * * p<0.001$

the late-pregnant rats compared with the unmated controls (Fig. 5).

Potential factors influencing the regulation of skeletalmuscle glycogen synthesis during pregnancy. Insulin resistance at the level of muscle glycogen synthesis has been linked to excessive exposure to lipids [27]. Fasting in late pregnancy leads to a pronounced switch to fat catabolism, much greater than occurs in non-pregnant animals over the same period [3]. To determine whether the altered muscle glycogen synthase responses in pregnancy reflected altered regulation of lipid availability, plasma NEFA and TAG concentrations and blood ketone body concentrations were measured in the unmated and pregnant groups before and after insulin stimulation. As anticipated, plasma TAG and blood ketone body concentrations after 24-h starvation were higher in the pregnant group [by 7.3-fold $(p<0.001)$ and 2.0-fold $(p<0.01)$, respectively; Fig. 6]. The concentrations of all three lipid fuels decreased in response to hyperinsulinaemia (Fig. 6). Systemic, circulating concentrations of plasma NEFA and TAG and blood ketone body concentrations did not differ significantly between the groups after 120-min exposure to insulin.

Recent studies have shown that hyperlactaemia increases muscle glycogen synthesis in vivo in resting rats $[28,29]$. This may be explained by an enhanced activity of glycogen synthase [29]. In the present study, basal lactate concentrations did not differ significantly between unmated and late-pregnant rats (Fig. 6). Hyperinsulinaemia increased circulating lac- 

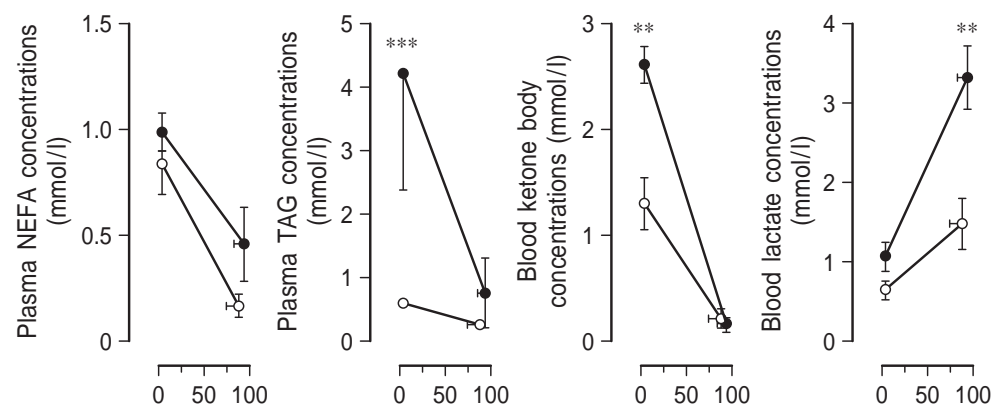

Plasma insulin concentration $(\mu \mathrm{U} / \mathrm{ml})$

Fig. 6. Effects of pregnancy on plasma NEFA, TAG and blood ketone body and lactate concentrations in the basal state and after euglycaemic insulin stimulation in unmated and 20-daypregnant rats. Further details are provided in Materials and methods. The results represent means \pm SEM for 5 unmated and 8 late-pregnant rats in the basal state and for 7 unmated and 5 late-pregnant rats in the insulin-stimulated state. Statistically significant differences between unmated and 20-daypregnant rats in either the basal or hyperinsulinaemic states are indicated by $* * p<0.01 ; * * * p<0.001$. Statistical analysis of the effects of hyperinsulinaemia is provided in the text

tate concentrations by 2.4 -fold $(p<0.05)$ in unmated rats and by 3.2 -fold $(p<0.01)$ in late-pregnant rats. As a result of the greater response of blood lactate concentrations to hyperinsulinaemia in the pregnant group after exposure to insulin, these concentrations were 2.3 -fold higher $(p<0.01)$ in the pregnant group compared with the unmated controls (Fig. 6).

\section{Discussion}

The control of the rate of glycogen synthesis from glucose in skeletal muscle involves several potential regulatory sites [11, 12]. These include glucose transport, hexokinase and glycogen synthase activity. Glycogen biosynthesis first requires the transport of glucose into muscle, a process stimulated by insulin $[3,30,31]$, and its subsequent phosphorylation to G6P. The relative contribution of the role of glycogen synthase activation in the stimulation of glycogen synthesis, however, remains controversial $[11,12]$. Both glucose uptake and glycogen concentrations are increased several fold in transgenic mice that overexpress GLUT 1 in skeletal muscle [32]. The activity of glycogen synthase is, however, unchanged [32], prompting the conclusion that glucose transport is the rate-limiting step for glycogen synthesis. The presence of higher glycogen concentrations in muscles of transgenic mice overexpressing an activated mutant form of glycogen synthase, in the absence of enhanced glucose uptake/phosphorylation, nevertheless suggested that glucose transport was not necessarily rate-limiting for glycogen synthesis. Thus, glycogen synthase may have an active role in determining glycogen accumulation [12].

We investigated the interrelation existing in vivos between stimulation of glucose uptake/phosphorylation and glycogen synthase activation in response to a rise in plasma insulin in the absence or presence of hyperglycaemia in unmated and late-pregnant rats after starvation. The studies were undertaken in 24-h starved rats since an extended antecedent period of starvation is necessary to observe relatively high rats of glycogen deposition when rats are subsequently refed [23]. In the presence of the allosteric activator G6P, full glycogen synthase activity is realised even with the most highly phosphorylated forms of glycogen synthase, and thus the \pm G6P activity ratio provides a kinetic index of the state of activation of the enzyme. In 24-h starved unmated rats, stimulation of glucose uptake/phosphorylation by increasing the plasma insulin concentration to approximately $35-40 \mu \mathrm{U} / \mathrm{ml}$ was statistically significant in each of the muscles studied but no significant activation of glycogen synthase was shown. A previous study examined the response of skeletal-muscle glycogen synthase to euglycaemic hyperinsulinaemia with plasma insulin at a threefold higher concentration [26]. As in this study, no significant activation of glycogen synthase was observed in slow-twitch skeletal muscle [26]. The greater degree of hyperinsulinaemia (achieved in the earlier study by using a higher rate of insulin infusion) did, however, elicit glycogen synthase activation in fast-twitch skeletal muscle [26]. Overall, results obtained with 24-h starved unmated rats suggest that regulation of glycogen deposition in fast-twitch skeletal muscle does not reside at the level of glycogen synthase activation when increases in circulating insulin concentrations are moderate, but possibly involve regulation of glycogen synthase when circulating insulin concentrations are higher. The present results do not, however, support an active role of glycogen synthase activation in regulating rates of glycogen accumulation in slow-twitch muscle of 24-h starved unmated rats in response to a rise in insulin alone. Evidence supporting this conclusion is provided by a study of the response of glycogen synthase to refeeding after starvation, where glycogen synthase dephosphorylation (assessed as G6P-inde- 
pendent activity) was not observed in association with glycogen storage in slow-twitch skeletal muscle [14].

Showing impaired glucose transport and phosphorylation by skeletal muscle in the basal (24-h starved) state and after euglycaemic hyperinsulinaemia in late pregnancy afforded a further opportunity to examine the potential relation between glucose phosphorylation and changes in glycogen synthase phosphorylation state in vivo. Steady-state glucose uptake/phosphorylation rates in skeletal muscles of the 24-h starved pregnant group during euglycaemic hyperinsulinaemia were consistently lower than in the $24-h$ starved unmated group. In addition, the increment in glucose utilisation elicited by hyperinsulinaemia was lower in 3 of the 5 skeletal muscles studied. In contrast, \% GSa was increased in two of the three fast-twitch skeletal muscles of the 24-h starved pregnant rats. In addition, \%GSa values for all three fast-twitch muscles after euglycaemic hyperinsulinaemia were higher than corresponding values in 24-h starved unmated rats. Pregnancy therefore does not impair but tends to enhance the response of glycogen synthase in fast-twitch muscle of 24-h starved rats to moderate hyperinsulinaemia. This is an important finding since the muscle mass of the rat consists mainly $(95 \%)$ of fast-twitch fibres [33]. Irrespective of the role of glycogen synthase activation in glycogen storage in pregnancy, the present results clearly show that the insulin resistance manifest at the level of glucose uptake/phosphorylation in 24-h starved pregnant rats does not extend to activation of glycogen synthase by insulin. This result argues for post-receptor mechanisms (which may include divergent signalling pathways) that modulate these individual downstream actions of insulin. This conclusion is strengthened by a study that has shown a clear-cut dissociation between the effect of insulin on the glycogen synthetic and glycolytic pathway in skeletal muscle [34].

Our study also shows rapid glycogen synthase activation in vivo in fast-twitch skeletal muscles by the short-term increases in circulating insulin and glucose concentrations induced by 15 -min glucose infusion after 24-h starvation. We have shown previously [26] that glycogen synthase responds to an acute rise in insulin after a lag period of approximately $5 \mathrm{~min}$, a period which is likely to reflect the time required for insulin to be transported across the vascular endothelial membrane [35]. A comparison between the increment in \% GSa elicited by euglycaemic hyperinsulinaemia and hyperglycaemic hyperinsulinaemia at similar insulin concentrations shows an effect $(p<0.05)$ of hyperglycaemia to promote glycogen synthase activation by insulin in fast-twitch muscle of unmated, but not late-pregnant, rats. As the \% GSa existing in fast-twitch muscles at the end of the infusions were similar in the two groups, it can be concluded that differences between 24-h starved pregnant and 24-h starved unmated rats in terms of the responses of glycogen synthase in fast-twitch skeletal muscle to hyperinsulinaemia alone are no longer evident when hyperglycaemia is also present (and insulin and glucose concentrations are matched between groups).

Whereas an effect of euglycaemic hyperinsulinaemia to activate glycogen synthase appears to be confined to the fast-twitch muscles, our study clearly shows rapid glycogen synthase activation in vivo in slow-twitch skeletal muscles by concomitant increases in insulin and glucose concentrations after 24-h starvation. The results indicate an essential role for hyperglycaemia in mediating glycogen synthase activation in slow-twitch muscle of 24-h starved rats. The effect of increased glycaemia to activate glycogen synthase in slow-twitch muscle is evident for both pregnant and unmated 24-h starved rats (cf. fast-twitch muscle) and the effect of hyperglycaemia is greater in SOL (and to a lesser extent in ADL) muscles of 24-h starved pregnant compared with 24-h starved unmated rats. It can be concluded that an increase in glycaemia facilitates activation of glycogen synthase by insulin in slow-twitch muscle of 24-h starved rats. In addition, pregnancy does not impair (and tends to enhance) activation of muscle glycogen synthase in response to insulin after 24-h starvation in both fast-twitch and slow-twitch muscle types, although increased glycaemia is prerequisite for the enhanced response in slow-twitch skeletal muscle to be shown.

We examined potential factors that might influence the regulation of skeletal-muscle glycogen synthase by insulin after 24-h starvation in pregnancy. In skeletal muscle, a negative feedback mechanism between tissue glycogen concentration and the rate of glycogen synthesis has been described [23, 24, 36]. In this study, the 24-h period of fasting before the study was performed resulted, however, in low muscle glycogen concentrations in both unmated and late-pregnant 24-h starved rats. There was no effect of pregnancy to accelerate glycogen depletion in response to starvation. The prevailing glycogen concentration cannot therefore account for differences observed in the responses of muscle glycogen synthase to insulin and glucose between unmated and late-pregnant 24-h starved rats. Late pregnancy is associated with accelerated and augmented production of lipid fuels during starvation (Fig. 6, [3]), an adaptation that spares glucose for use by the feto-placental unit by limiting maternal glucose utilisation $[2,3,5]$. The possibility therefore exists that altered exposure to lipid fuels impacts on the regulation of glycogen synthase in late pregnancy. Two studies have recently addressed the issue of the effects of prolonged increases in lipid exposure on glycogen synthase activity. Both studies reported that glycogen synthesis rates and glycogen synthase activities in muscle were decreased in response to prolonged (5-h) increased plasma NEFA 
concentration in unmated rats (induced by intravenous triglyceride/heparin infusion) $[37,38]$. In our study, glycogen synthase activities after 24-h starvation were unaffected in late-pregnant rats compared with unmated rats, despite an augmented lipid-fuel supply in the former group. Furthermore, the response of muscle glycogen synthase to hyperinsulinaemia in the absence or presence of glycaemia was not impaired (and in some cases was enhanced) by late pregnancy. Thus, a modified response of muscle glycogen synthase to insulin in 24-h starved pregnant rats occurs despite increased exposure to lipid fuels. A recent study has shown that a rise in the circulating lactate concentration enhances glycogen synthase activity [29]. Given that lactate concentrations after insulin stimulation were higher in the pregnant group, our results are compatible with the suggestion [29] that lactate participates in the regulation of skeletalmuscle glucose disposal. We therefore propose that hyperlactaemia during hyperinsulinaemia after 24-h starvation assists muscle glycogen storage during late pregnancy through enhancing glycogen synthase activity and thereby compensates for insulin resistance at the level of glucose uptake and phosphorylation.

In summary, our study shows that glycogen synthase activation in skeletal muscle in response to hyperinsulinaemia in the absence or presence of hyperglycaemia after 24-h starvation is not impaired, and may be enhanced, during late pregnancy. Within the physiological context, an enhanced response of glycogen synthase to insulin in slow-twitch muscle is critically dependent on the extent to which glycaemia is concomitantly raised. Although glycogen synthase activation is not impaired, late pregnancy is nevertheless associated with reduced muscle glucose uptake and phosphorylation, arguing for post-receptor modulation of these individual downstream actions of insulin. The dissociation between the efficacy of the effects of insulin to stimulate insulin-dependent glucose uptake and facilitate glycogen synthase activation in muscle ensures that available circulating glucose is preferentially directed towards the fetus, rather than towards the mother, under conditions of moderate hyperinsulinaemia (as would be observed during the initial phase of refeeding after starvation) but that maternal muscle glycogen storage is minimally compromised.

Acknowledgements. This study was supported in part by funds from the Juvenile Diabetes Foundation International (No 194179) and the European Commission (Biomed 2 Programme) (No BMH4-CT97-2717). We are grateful to L. Fryer for assistance with the insulin and glycogen synthase assays.

\section{References}

1. Holness MJ, Changani KK, Sugden MC (1991) Progressive suppression of muscle glucose utilization during pregnancy. Biochem J 280: 549-552

2. Nolan CJ, Proietto J (1994) The feto-placental glucose steal phenomenon is a major cause of maternal metabolic adaptation during late pregnancy in the rat. Diabetologia 37: 976-984

3. Holness MJ, Sugden MC (1997) Glucoregulation during progressive starvation in late pregnancy in the rat. Am J Physiol 272: E556-E561

4. Leturque A, Ferré P, Burnol A-F, Kandé J, Maulard P, Girard J (1986) Glucose utilization rates and insulin sensitivity in vivo in tissues of virgin and pregnant rats. Diabetes 35: 172-177

5. Leturque A, Burnol A-F, Ferré P, Girard J (1984) Pregnancy-induced insulin resistance in the rat: assessment by glucose clamp technique. Am J Physiol 246: E25-E31

6. Holness MJ, Sugden MC (1993) Changes in rates of glucose utilization and regulation of glucose disposal by fast-twitch skeletal muscles in late pregnancy. Biochem J 292: 431-438

7. Sugden MC, Holness MJ (1994) The role of the glucose/fatty acid cycle in the selective modulation of non-oxidative and oxidative glucose disposal by oxidative muscle in late pregnancy. Biol Chem Hoppe Seyler 375: 141-147

8. Rossetti L, Giaccari A (1990) Relative contribution of glycogen synthesis and glycolysis to insulin-mediated glucose uptake. A dose-response euglycemic clamp study in normal and diabetic rats. J Clin Invest 85: 1785-1792

9. Shulman GI, Rothman DL, Jue T, Stein P, DeFronzo RA, Shulman RG (1990) Quantitation of muscle glycogen synthesis in normal subjects and subjects with non-insulin-dependent diabetes by $13 \mathrm{C}$ nuclear magnetic resonance specroscopy. New Engl J Med 322: 223-228

10. Thiébaud D, Jacot E, DeFronzo RA, Maeder E, Jéquier E, Felber JP (1982) The effect of graded doses of insulin on total glucose uptake, glucose oxidation, and glucose storage in man. Diabetes 31: 957-963

11. Holness MJ, Fryer LGD, Sugden MC (1997) Endocrine and nutritional modulation of glucose disposal and storage in muscle. Biochem Soc Trans 25: 1-7

12. Lawrence JC, Roach PJ (1997) New insights into the role and mechanism of glycogen synthase activation by insulin. Diabetes 46: 541-547

13. Lawrence JC (1992) Signal transduction and protein phosphorylation in the regulation of cellular metabolism by insulin. Annu Rev Physiol 54: 177-193

14. James AP, Flynn CB, Jones SL, Palmer TN, Fournier PA (1998) Re-feeding after starvation involves a temporal shift in the control site of glycogen synthesis in rat muscle. Biochem J 329: 341-347

15. Lederman S, Rosso P (1981) Effects of fasting during pregnancy on maternal and fetal weight and body composition in well-nourished and undernourished rats. J Nutr 111: 1823-1832

16. Ariano MA, Armstrong RB, Egerton VR (1973) Hindlimb muscle fiber populations of five animals. J Histochem $\mathrm{Cy}$ tochem 21: 51-55

17. Holness MJ, Sugden MC (1990) Glucose utilization in heart, diaphragm and skeletal muscle during the fed-tostarved transition. Biochem J 270: 245-249

18. Ferré P, Leturque A, Burnol A-F, Pénicaud L, Girard J (1985) A method to quantify glucose utilization in vivo in skeletal muscle and white adipose tissue of the anaesthetized rat. Biochem J 228: 103-110 
19. Holness MJ, Sugden MC (1996) Suboptimal protein nutrition in early life later influences insulin action in pregnant rats. Diabetologia 39: 12-21

20. Nimmo HG, Proud CG, Cohen P (1976) The phosphorylation of rabbit skeletal muscle glycogen synthase by glycogen synthase kinase- 2 and adenosine- $3^{\prime}: 5^{\prime}$-monophosphate dependent protein kinase. Eur J Biochem 68: 31-44

21. Thomas JA, Schlender KK, Larner J (1968) A rapid filter paper assay for UDP-glucose-glycogen glucosyltransferase, including an improved biosynthesis for UDP(14C)glucose. Anal Biochem 25: 486-499

22. Holness MJ, Howard RM, Sugden MC (1992) Glucose utilization and disposition by skeletal muscle during unrestricted feeding. Biochem J 286: 395-398

23. Holness MJ, Sugden MC (1991) Glucose disposal by skeletal muscle in response to re-feeding after progressive starvation. Biochem J 277: 429-433

24. Furler SM, Goldstein M, Cooney GJ, Kraegen EW (1998) In vivo quantification of glucose uptake and conversion to glycogen in individual muscles of the rat following exercise. Metabolism 47: 409-414

25. Larner J, Villar-Palasi C (1971) Glycogen synthase and its controls. Curr Top Cell Regul 3: 195-236

26. Sugden MC, Holness MJ, Fryer LGD (1997) Differential regulation of glycogen synthase activity by insulin and glucose in vivo in individual skeletal muscles of the rat. Am J Physiol 273: E479-E487

27. Oakes ND, Cooney GJ, Camilleri S, Chisholm DJ, Kraegen EW (1997) Mechanisms of liver and muscle insulin resistance induced by chronic high-fat feeding. Diabetes 46 : 1768-1774

28. Ryan C, Radziuk J (1995) Distinguishable substrate pools for muscle glyconeogenesis in lactate-supplemented recovery from exercise. Am J Physiol 269: E538-E550

29. Pagano C, Granzotto M, Giaccari A et al. (1997) Lactate infusion to normal rats during hyperglycemia enhances in vivo muscle glycogen synthesis. Am J Physiol 273: R2072-R2079

30. James DE, Jenkins AB, Kraegen EW (1985) Heterogeneity of insulin action in individual muscles in vivo: euglycemic clamp studies in rats. Am J Physiol 248: E567-E574

31. Baron AD, Brechtel G, Wallace P, Edeman SV (1988) Rates and tissue sites of non-insulin- and insulin-mediated glucose uptake in humans. Am J Physiol 255: E769-E774

32. Ren JM, Marshall BA, Gulve EA et al. (1993) Evidence from transgenic mice that glucose transport is rate-limiting for glycogen deposition and glycolysis in skeletal muscle. J Biol Chem 268: 16113-16115

33. Armstrong RB, Phelps RO (1984) Muscle fiber type composition of the rat hindlimb. Am J Anat 171: 259-272

34. Koopmans SJ, Mandarino L, DeFronzo RA (1998) Time course of insulin action on tissue-specific intracellular glucose metabolism in normal rats. Am J Physiol 274: E642-E650

35. Hachiya HL, Halban PA, King GL (1988) Intracellular pathways of insulin transport across vascular endothelial cells. Am J Physiol 255: C459-C464

36. Munger RE, Temler E, Jallut D, Haesler E, Felber JP (1993) Correlations of glycogen synthase and phosphorylase activities with glycogen concentration in human muscle biopsies. Evidence for a double-feedback mechanism regulating glycogen synthesis and breakdown. Metabolism 42: $36-43$

37. Chalkley SM, Hettiarachchi M, Chisholm DJ, Kraegen EW (1998) Five-hour fatty acid elevation increases muscle lipids and impairs glycogen synthesis in the rat. Metabolism 47: $1121-1126$

38. Park JY, Kim CH, Hong SK, Suh KI, Lee KU (1998) Effects of FFA on insulin-stimulated glucose fluxes and muscle glycogen synthase activity in rats. Am J Physiol 275: E338-E344 